\title{
Intra-lacrimal Sustained Delivery of Rapamycin Shows Therapeutic Effects Without Systemic Toxicity in a Mouse Model of Autoimmune Dacryoadenitis Characteristic of Sjögren's Syndrome
}

Yaping Ju', Maria C. Edman², Hao Guo', Srikanth Reddy Janga², Santosh Peddi', Stan

G. Louie ${ }^{3}$, Jason A. Junge ${ }^{4}$, J. Andrew MacKay ${ }^{1,2,5^{*}}$, and Sarah F. Hamm-A/varez ${ }^{1,2^{*}}$

${ }^{1}$ Department of Pharmacology and Pharmaceutical Sciences, School of Pharmacy,

University of Southern California, Los Angeles, California, United States

${ }^{2}$ Department of Ophthalmology, Roski Eye Institute, Keck School of Medicine, University

of Southern California, Los Angeles, California, United States

${ }^{3}$ Department of Clinical Pharmacy, School of Pharmacy, University of Southern California,

Los Angeles, California, United States

${ }^{4}$ Department of Biological Sciences, Translational Imaging Center, Dornsife College of

Letters, Arts, and Sciences, University of Southern California, Los Angeles, California

90089, United States 
${ }^{5}$ Department of Biomedical Engineering, Viterbi School of Engineering, University of

Southern California, Los Angeles, California 90089, United States

${ }^{*}$ Co-corresponding authors

A

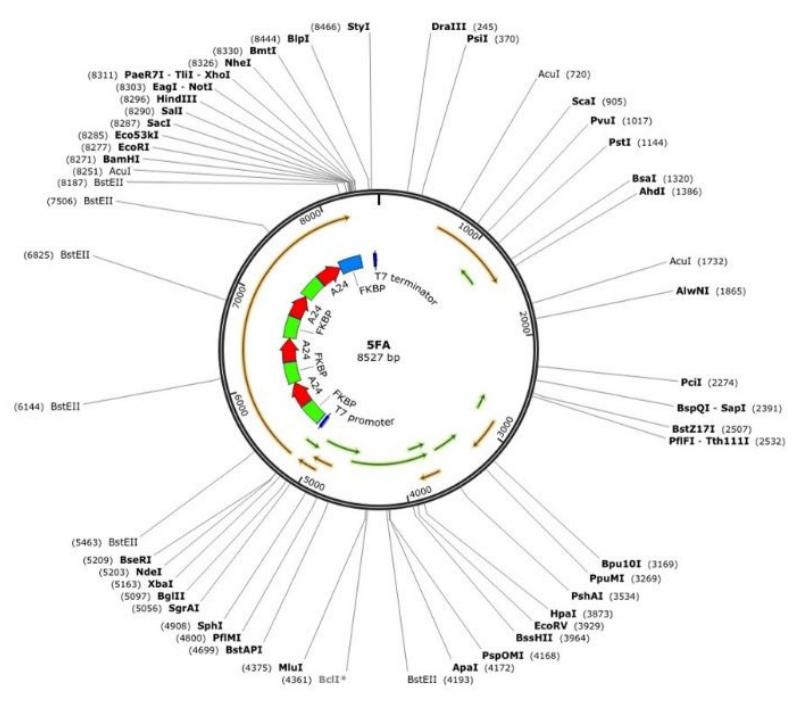

B

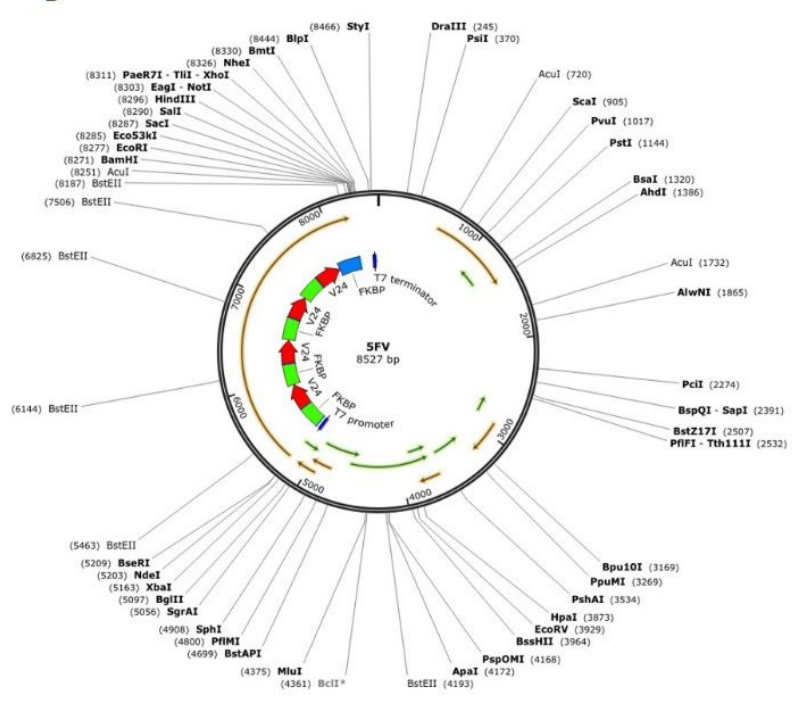

Fig S1. Cloning schematic of A) 5FA and B) 5FV.

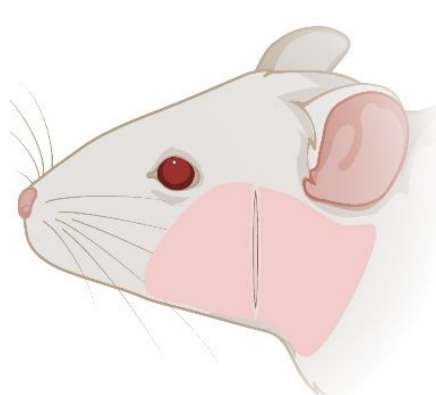

1. Shave the cheek, and make a small cut between the eye and the ear.

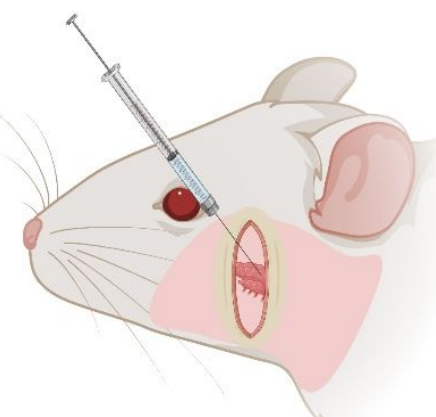

2. Locate the lacrimal gland, and inject.

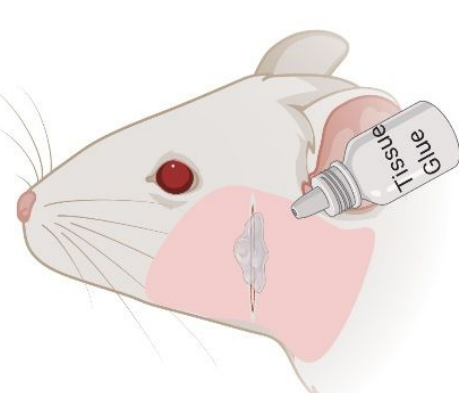

3. Close the surgery cut with tissue glue. 
Fig S2. Schematic illustration of the process of intra-LG gland injection.
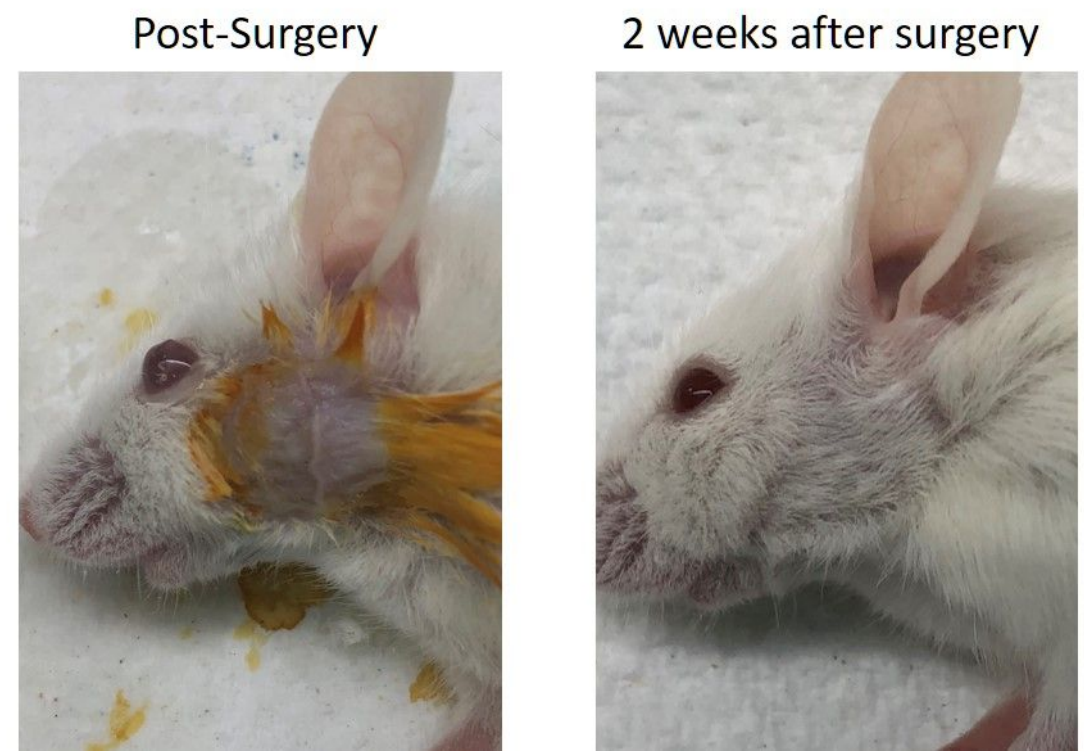

Fig S3. Evaluation of the surgical cut after intra-LG injection. The surgical cut was healed completely after 2 weeks. 
A

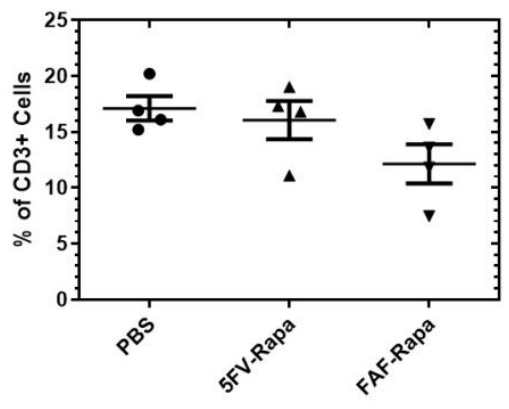

D

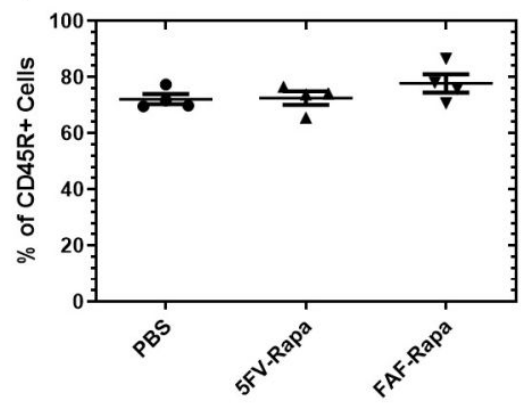

B

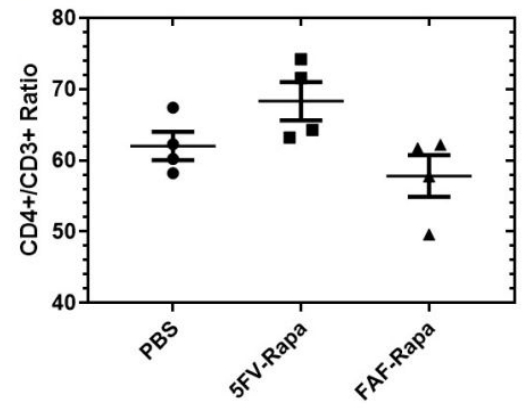

E

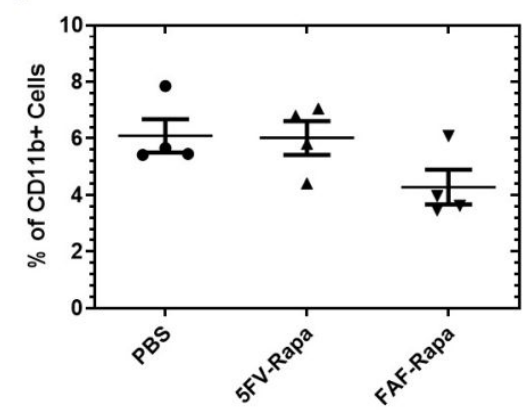

C

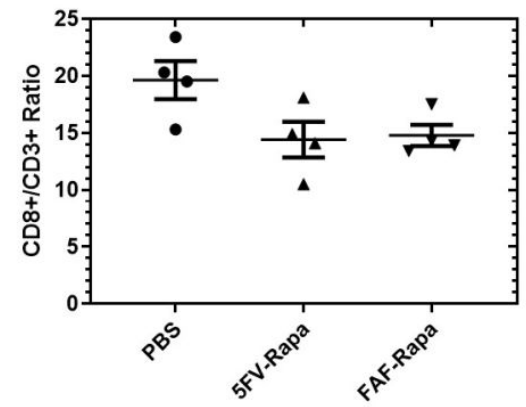

Figure S4. Flow cytometry analysis of lymphocyte composition in the LG after treatments reveals no significant changes in the profile of infiltrating cells. A) Percentage of CD3+ cells relative to total lymphocytes isolated. B). Percentage of CD4+ cells within the total CD3+ cells. C). Percentage of CD8+ cells within the total CD3+ cells. D). Percentage of CD45R+ relative to total lymphocytes isolated. E) Percentage of CD11b+ cells relative to total lymphocytes isolated. $\mathrm{n}=4$ mice for each group. 


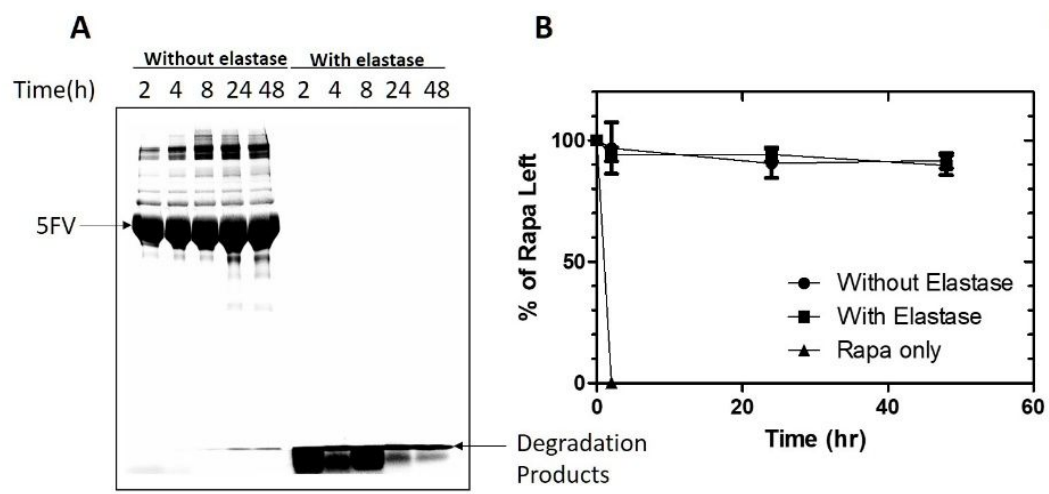

C

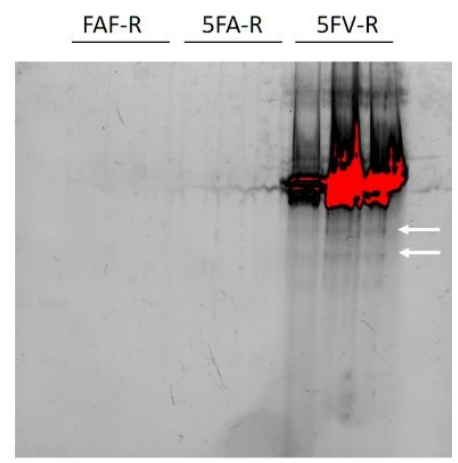

Figure S5. In Vitro degradation and In Vivo retention of ELP-FKBP. Rhodamine-labeled 5FV-Rapa, with or without elastase, was dialyzed under sink conditions against PBS at $37^{\circ} \mathrm{C}$. A) The mixture from the dialysis cassette was collected $2 \mathrm{~h}, 4 \mathrm{~h}, 8 \mathrm{~h}, 24 \mathrm{~h}$ and 48 $\mathrm{h}$ after dialysis and resolved by SDS-PAGE to assess the protein degradation. B) Rapa concentration was assessed by HPLC at $2 h, 24 h$, and $48 \mathrm{~h}$ after dialysis $(n=2) . C)$ NOD mice were treated with rhodamine-labeled FAF-Rapa subcutaneously $(180 \mu \mathrm{M}$ Rhodamine, $100 \mu \mathrm{l})$, 5FA-Rapa intraLG (300 $\mu \mathrm{M}$ Rhodamine, $10 \mu \mathrm{l}), 5 \mathrm{FV}-$ Rapa intraLG (300 $\mu \mathrm{M}$ Rhodamine, $10 \mu \mathrm{l}$ ) comparably to the efficacy study. LG were collected $24 \mathrm{~h}$ after treatment and lysates resolved by SDS-PAGE $(n=3)$. White arrows, possible degradation products. 


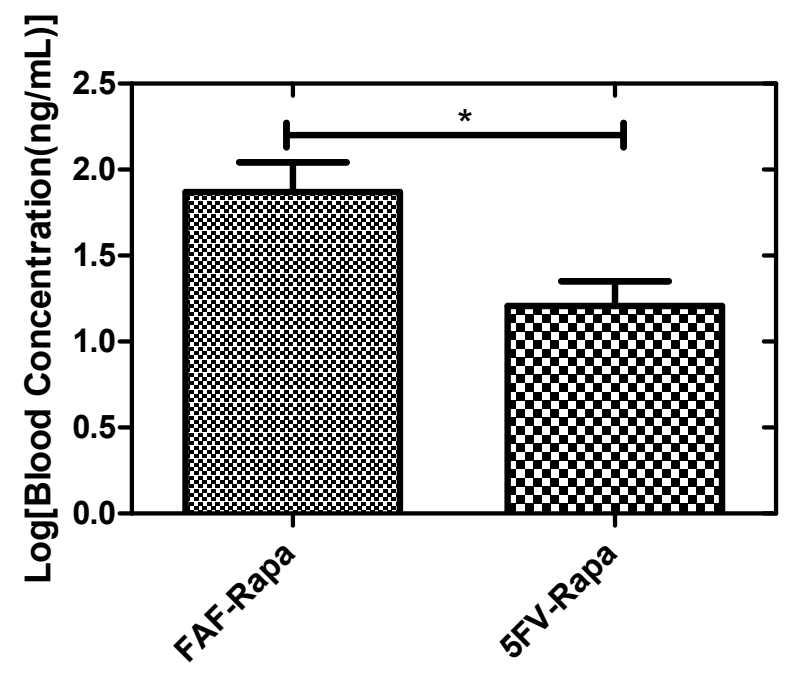

Figure S6. Amount of Rapa in the blood 24h after treatment. NOD mice aged $16 \mathrm{w}$ was treated with FAF-Rapa subcutaneously $(1 \mathrm{mg} / \mathrm{kg}), 5 F V-R a p a$ intraLG $(0.44 \mathrm{mg} / \mathrm{kg})$ in the same way as the efficacy study. Even after a single dose, the intraLG treatment yielded an $\sim 5$ fold decrease in plasma Rapa, which may explain the reduction of observed side effects. Blood was collected one day after injection and amount of Rapa is measured by $\operatorname{LC} / M S(n=4) .{ }^{*} p<0.05$. 


\section{Flow cytometry}

LG from individual mice from the therapeutic study were collected and digested with

multiple rounds of incubation in Hank's-EDTA medium and collagenase/hyaluronidase/DNAse cocktail as previously described ${ }^{1}$. Supernatants were collected and passed through a $10 \mu \mathrm{m}$ cell strainer. Cells were pelleted and resuspended in cell-staining media at a concentration of $15 \times 10^{6} \mathrm{cells} / \mathrm{mL}$. Cells were treated with mouse TruStain FcX Plus ( $1 \mu \mathrm{g}$ per $10^{6}$ cells) for $10 \mathrm{~min}$ to block Fc-receptors. LIVE/DEAD fixable aqua dead cell stain was added to the cell suspension $\left(1 \mu 1\right.$ per $10^{6}$ cells) followed by incubation on ice for $30 \mathrm{~min}$. Fluorescently-conjugated antibodies including anti-CD45R, anti-CD11b, anti-CD3, anti-CD4, anti-CD8 were added to the cell suspension at optimized concentrations to label B cells, Macrophages, T cell, CD4+ T cells and CD8+ $T$ cells respectively, and incubated on ice in the dark for 30 min. Cells were washed twice, centrifuged at $350 \times \mathrm{g}$ for $5 \mathrm{~min}$, and resuspended in cell-staining buffer for flow analysis on a BD LSR Fortessa X20 (Franklin Lakes, NJ, USA). Unstained live cells were used as controls to identify positive staining. 
1. Meng, Z.; Klinngam, W.; Edman, M. C.; Hamm-Alvarez, S. F., Interferon-Y treatment in vitro elicits some of the changes in cathepsin $S$ and antigen presentation characteristic of lacrimal glands and corneas from the NOD mouse model of Sjögren's Syndrome. PloS one 2017, 12, (9), e0184781. 